\section{Unknown syndrome: mental retardation with postaxial polydactyly, congenital absence of hair, severe seborrhoeic dermatitis, and Perthes' disease of the hip}

SUMmaRY We report a six year old male with mental retardation, postaxial polydactyly and syndactyly, atrichia congenita totalis, severe seborrhoeic dermatitis, recurrent staphylococcal skin sepsis, and Perthes' disease of the hip. His birth may have resulted from an incestuous mating.

\section{History}

The child's mother was a 22 year old educationally subnormal primigravida, and the father was her 46 year old stepfather, who may have been her natural father. Her half brother had died at birth with malformations, and two half sisters and a half brother, all of whom were fathered by her stepfather, were educationally subnormal. There was a strong history of atopy in the stepfather's family. The pregnancy proceeded normally, and the patient was born by normal spontaneous vaginal delivery at term, weighing $2.95 \mathrm{~kg}$.

\section{Medical history}

At birth he was noted to have complete atrichia witl absence of eyelashes and eyebrows. There was postaxial polydactyly with six toes on each foot and syndactyly of the second and third toes (fig 1). On the left hand there were six digits, but on the right there were only four, a thumb and three fingers (fig 2). A haemangioma of the scalp was: present in the right parietal region. His penis was smallo but the testes were descended. He subsequently developed a severe seborrhoeic exfoliative dermatitis with hypos albuminaemia and hepatosplenomegaly which respondef to treatment with a combination of topical steroids and systemic antistaphylococcal agents, as recommended fo? the treatment of Leiner's disease. On stopping maing tenance anti-staphylococcal therapy staphylococcal skim infections occurred on two occasions. His development was delayed and he had epilepsy. At five years he began te. limp and hip $x$ ray showed avascular necrosis of both femoral heads, with an associated metaphyseal reactionळ At six years of age he remains hairless with very rough skifo (fig 3a). His teeth and nails are normal. The nose ig rather beaked with a prominent columella, and the left ea? has an irregularly folded helix (fig $3 \mathrm{~b}$ ). The head circum? ference is normal and his height is above the 3rd centil\& and weight above the 75th centile.

\section{Investigations}

Chromosome examination with $\mathrm{G}$ banding showed

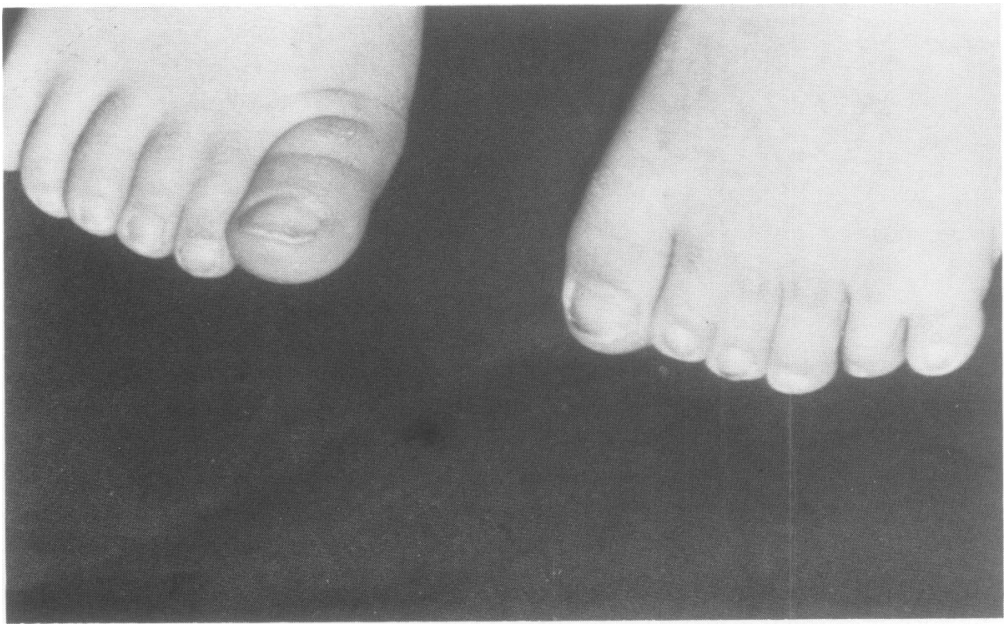

Received for publication 6 April 1987 Accepted for publication 7 April 1987 FIG 1 Feet at 22 months showing
postaxial polydactyly and partial syndactyly of the second and third toes. 

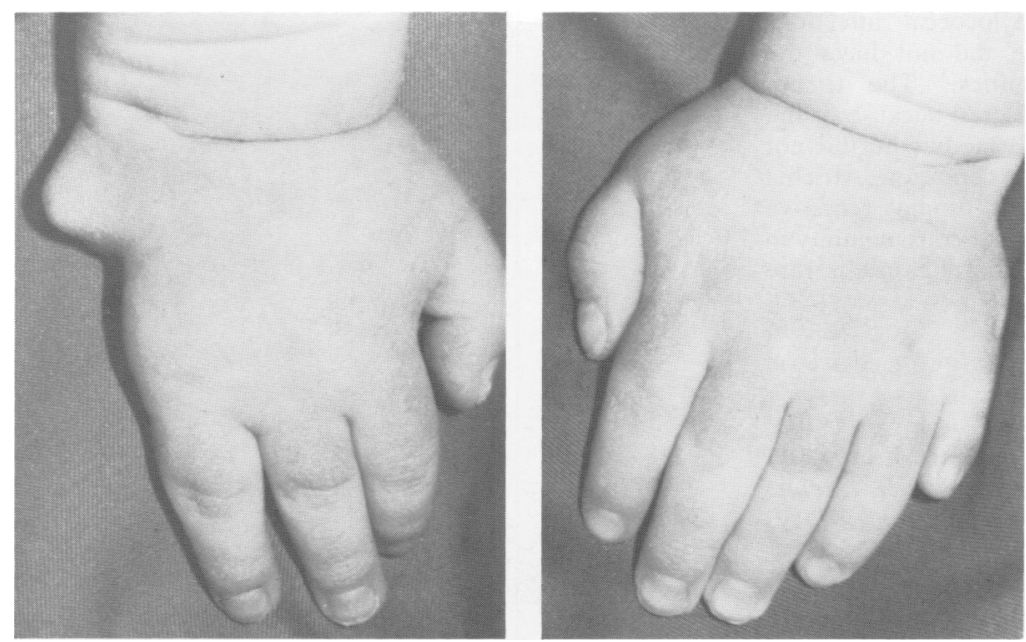

FIG 2 Hands at 22 months showing postaxial polydactyly on the left and absent little finger on right hand.

normal male karyotype, 46,XY. Immunological studies, including immunoglobulins, complement, and yeast opsonisation, were all normal. CT scan of the head showed slightly dilated ventricles. $X$ rays of the hips showed fragmentation and increased density of the femoral heads with some changes in the femoral necks. The findings were asymmetrical and were consistent with Perthes' disease.

\section{Discussion}

The combination of features in this patient has not previously been described. The skin abnormalities suggest a form of ectodermal dysplasia, but he has normal nails and teeth, and the polydactyly and Perthes' disease could not be explained by this. The severe seborrhoeic dermatitis
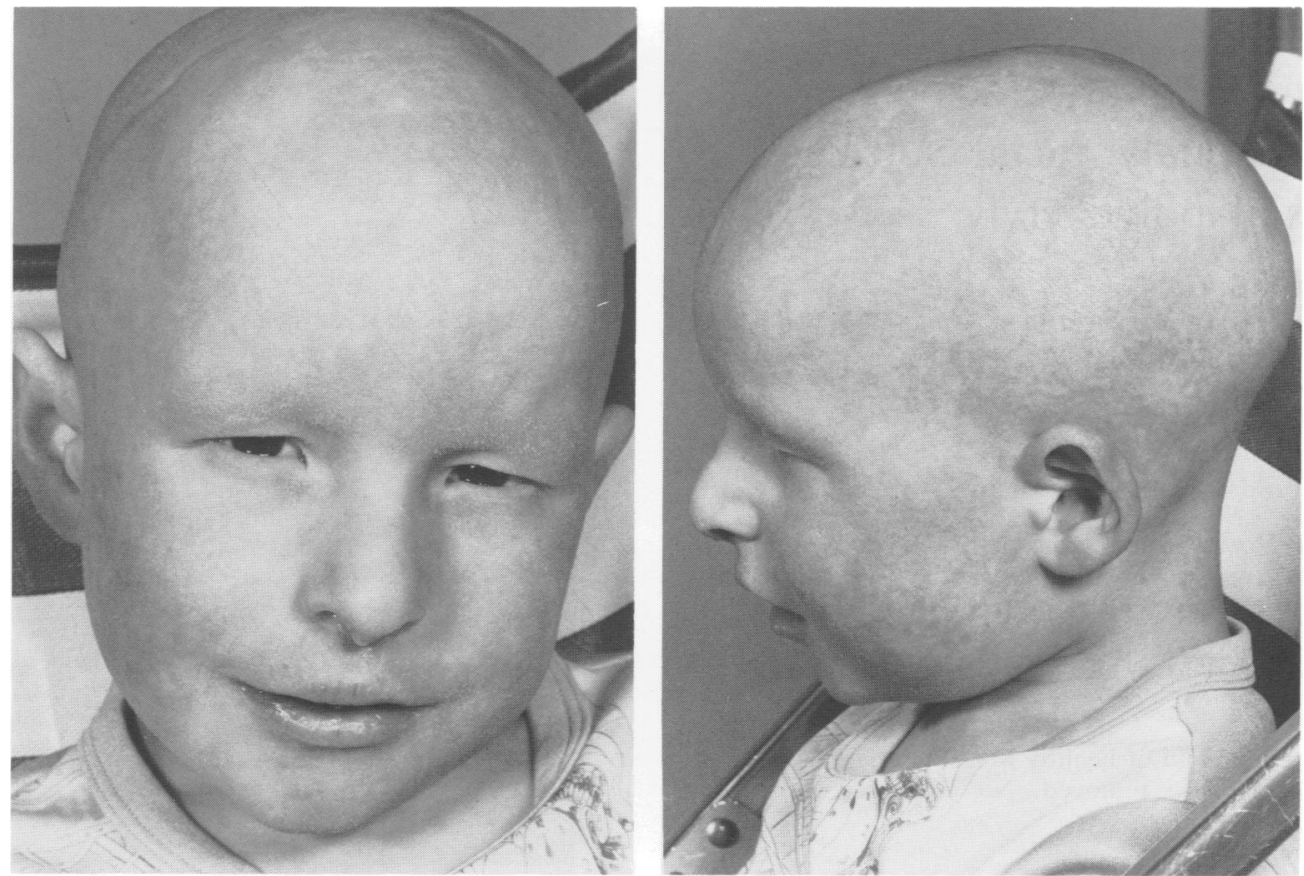

FIG 3 (a) Facial appearance at five years. Note complete absence of hair. (b) Lateral view of face at five years. 
with hypoalbuminaemia and staphylococcal infections resembled Leiner's disease, but he did not have the associated immunological abnormalities. ${ }^{1}$ The trichorhinophalangeal syndrome was considered because of the sparse hair and femoral head dysplasia, but he did not show the other skeletal changes of this condition. Atrichia congenita may be inherited as an autosomal recessive disorder ${ }^{2}$ and in view of the possibility of consanguinity in the child's family, it seems likely that he has a rare autosomal recessive condition.

We should like to thank Dr Andrew Warin for advice on the dermatological aspects of this case.

C Garrett AND J H TRIPP Koyal Devon and Exeter Hospital (Wonford), Exeter, $E X 25 D W$.

\section{References}

' Jacobs JC, Miller ME. Familial Leiner's discase: a deficiency of the opsonic activity of serum complement. Pediatrics 1972;46: 225-32.

2 Cantu JM, Sanchez-Corona J, Gonzalez-Mendosa A, Martinez R. Garcia-Cruz D. Autosomal recessive inheritance of atrichia congenita. Clin Genet 1980;117:209-12.

Correspondence and requests for reprints to $\mathrm{Dr}$ C Garrett, Department of Clinical Genetics, Royal Devon and Exeter Hospital (Wonford), Barrack Road, Exeter EX2 5DW.

\section{Unknown syndrome: microcephaly, facial clefting, and preaxial polydactyly}

SUMMARY We present a four year old boy with short stature, disproportionate microcephaly, developmental delay, convulsions, bilateral cleft lip and palate, and bifid right thumb.

\section{History}

Prenatal. No drugs in pregnancy. Maternal pyrexia for 24 hours at five weeks due to flu-like illness. Normal intrauterine movements.

Birth. Breech delivery at term. Birth weight $2.07 \mathrm{~kg}(<3 \mathrm{rd}$ centile).

Family. Only child of healthy Mauritian parents who are first cousins once removed. No other relevant family history.

Received for publication 18 June 1987. Accepted for publication 25 June 1987.

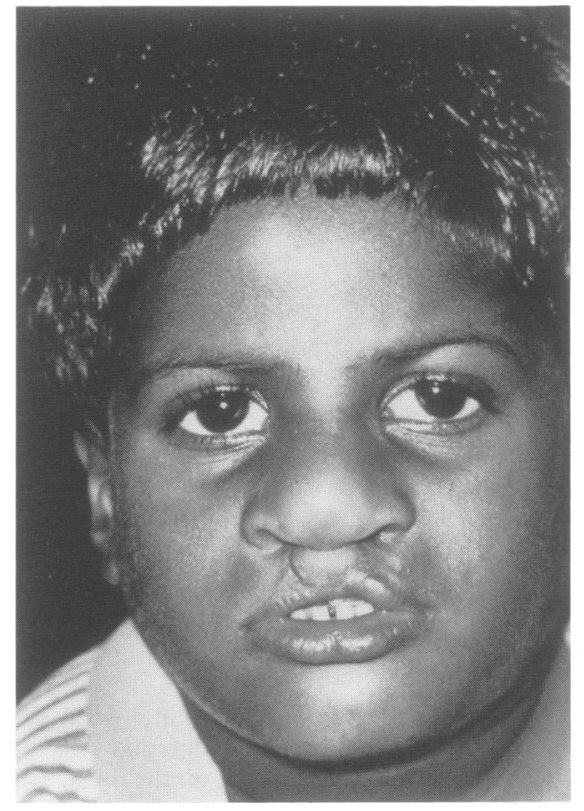

FIG 1 Facial view of the patient aged four years.

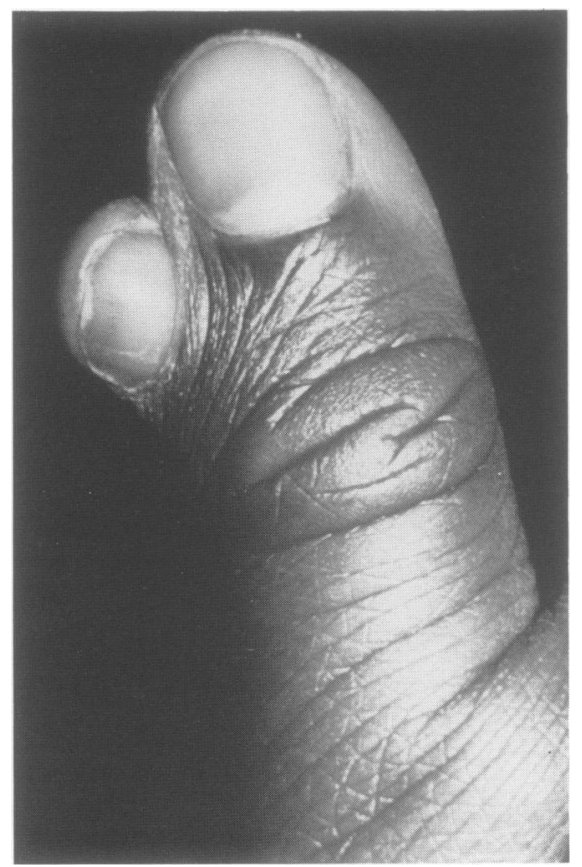

FIG 2 View of the patient's right thumb. 